\title{
Guest Editorial: Large-scale Data Management for Mobile Applications
}

\author{
Thierry Delot ${ }^{1}$ - Sandra Geisler ${ }^{2}$. \\ Sergio Ilarri ${ }^{3}$. Christoph Quix ${ }^{4}$
}

Published online: 23 May 2015

(C) Springer Science+Business Media New York 2015

The increasing functionality and capacity of mobile devices have enabled new mobile applications which require new approaches for data management. Users want to have a seamless integration of their data on their mobile with other devices, which can be either classical devices such as a desktop PC or other mobile devices. On the one hand, the growing computing power of mobile devices and the availability of "Big Data" to mobile users facilitate the development of powerful mobile applications. On the other hand, the limitations of mobile devices with respect to energy, storage, display size, communication bandwidth, and real-time capabilities have to be considered. Due to the growing volume of the data that has to be managed, the availability of huge datasets, the emergence of non-traditional techniques for data management (e.g., NoSQL systems), and the spreading of cloud computing, new efforts are expected in this area.

Information management in mobile applications is a complex problem space which requires the consideration of the aforementioned constraints. Under the umbrella of

Sergio Ilarri

silarri@unizar.es

Thierry Delot

tdelot@univ-valenciennes.fr

Sandra Geisler

geisler@dbis.rwth-aachen.de

Christoph Quix

christoph.quix@fit.fraunhofer.de

1 University of Valenciennes \& Inria Lille, Valenciennes, France

2 RWTH Aachen University, Aachen, Germany

3 University of Zaragoza, Zaragoza, Spain

4 Fraunhofer FIT, Sankt Augustin, Germany 
large-scale data management, a variety of hot topics could be considered, such as: Big Data in mobile applications, data stream management in mobile environments, management and processing of geospatial data, and location-based services. This special issue aimed at publishing recent, outstanding research results about technologies and mechanisms, which support the management of mobile, complex, integrated, distributed, and heterogeneous data-focused applications at a large scale. We invited research papers as well as papers which address challenges in developing industrial-strength mobile applications that need to manage a considerable amount of data. After a rigorous selection process, four papers were finally accepted:

- Physical Design for Distributed RFID-based Supply Chain Management, by Roberto De Virgilio and Franco Milicchio, tackles the physical design of a scalable system that distributes the workload of processing radio-frequency identification (RFID) data streams. The system proposed is based on a general framework for supply chain management, which exploits principles of the tensorial calculus.

- Managing Big Data Experiments on Smartphones, by Georgios Larkou, Marios Mintzis, Panayiotis G. Andreou, Andreas Konstantinidis, and Demetrios Zeinalipour-Yazti, presents an approach to transparently manage the complexity of large-scale data management experiments on real smartphones through an intuitive web-based interface. Moreover, the authors apply the proposed architecture, called SmartLab, to the case study of an indoor positioning system called Anyplace.

- SODA: A Framework for Spatial Observation Data Analysis, by Sebastáin Villarroya, José R.R. Viqueira, Manuel A. Regueiro, José A. Taboada, and José M. Cotos, presents SODA (Spatial Observation Data Analysis), a framework supporting spatio-temporal declarative analysis over large warehouses of geospatial observation data. The proposal is qualitatively evaluated and compared with other data management technologies and approaches, and some issues concerning its ongoing implementation are also discussed.

- Merging Sub-Networks in Self-Managed Vehicular Ad-hoc Networks, by Cándido Caballero-Gil, Pino Caballero-Gil, and Jezabel Molina-Gil, concerns aspects related to data management in the context of Vehicular Ad-Hoc Networks (VANETs). Specifically, the authors tackle the problem of merging sub-networks in VANETs compliant with the IEEE 802.11 standard. Two solutions are proposed: a deterministic solution and a proposal based on fuzzy logic.

We would like to thank all the authors for their valuable contribution to this special issue, as well as the reviewers for their great efforts in providing timely and insightful reviews. We believe that the papers selected are a good representative of the efforts being performed in the field and we hope that they will encourage further research in the area. The guest editors would also like to thank the support of some projects that fund their research activities in relation to the topic of the special issue (TIN201346238-C4-4-R, DGA-FSE, UMIC Research Cluster at RWTH Aachen University).

Thierry Delot, Sandra Geisler, Sergio Ilarri, Christoph Quix Guest Editors 